\title{
A NEW TRIPLE OPTIMALITY CRITERION CDKL
}

\author{
M. M. Seyam \\ m.seyam@science.tanta.edu.eg
}

Faculty of Science, Tanta University

Received 7/3/2018

Revised 19/3/2018

Accepted 22/5/2018

\begin{abstract}
In this paper, three optimality criteria will be compounded to give a new criterion, namely, CDKL-optimality for parameter estimation, estimating the area under curve and model discrimination for any kind of regression models, with homoscedastic or herteroscedastic errors, which may be Gaussian or not. CDKL-compound criterion is proposed for copula models.
\end{abstract}

Keywords: C-, D-, KL-, CD-, DKL- and CDT-optimum; Equivalence theorem; copula model.

MSC: 62-XX, 62Kxx, $62 \mathrm{~K} 05$.

\section{1- Introduction}

The optimal design theory was firstly introduced by Smith [1], some optimality criteria are proposed by Atkinson et al. [2]. C-optimality is presented by Elfving [3] which supplied a geometrical interpretation to obtain C-optimal designs. Dette and Holland-Letz [4] proposed Coptimal designs for heteroscedastic regression by using a geometric characterization. The most important criterion for parameter estimation is D-optimality, announced by Wald [5], numerous publications on D-optimality can be seen in Atkinson et al. [2]. Atkinson [6,7] proposed Toptimality for discriminating between two rival models and between several models. In order to act towards any distribution for the random errors see, López-Fidalgo et al. [8,9], which proposed KL-optimality criterion based on the Kullback-Liebler distance. A generalization for the KLoptimality criterion demonstrated by Tommasi [10], to deal with discrimination amongst different non-normal models.

In this paper, the aim is to associate the three-optimality criterion in one design criterion for model discrimination, parameter estimation and estimating the area under curve, namely, CDKL-optimality criterion. CDT-optimality criterion introduced by Abd El-monsef and Seyam 
[11] which is only for regression models, but the proposed criterion named by CDKL-optimality can be applied for nested regression models or not. Moreover, it has suggested for copula models.

During a long time, statisticians have been concerned with the connection between a multivariate distribution function and its lower dimensional margins. Sklar [12] gave the answer to this situation for univariate marginal case by forming a new class of functions is called copulas. In many areas of applied statistics copula models becomes a standard tool, such as, medicine Nikoloulopoulos and Karlis [13], marketing Danaher and Smith [14] and time series analysis Patton [15].

This paper is prepared as follows: C-, D- and KL-optimum designs are presented in Section 2 respectively. DKL - and CD-optimum designs is reviewed in Section 3. A new criterion namely CDKL-optimality is derived in Section 4. In Section 5, CDKL-criterion is proposed for copula models.

\section{2- C-, D-, KL-optimum designs}

\section{$2.1 \mathrm{KL}$-optimum design}

López-Fidalog et al. [9] proposed the KL-criterion which discriminate between models with normally distributed observations. It was applied to discriminate between the popular Michaelis-Menten model besides an extension of it under the lognormal and gamma distributions.

Let $f_{1}\left(y, x, \theta_{1}\right)$ and $f_{2}\left(y, x, \theta_{2}\right)$ be two rival density functions, which depends on an experimental condition $x \in \mathcal{X}$ and $\theta_{i} \in \Omega_{i} \subset \mathbb{R}^{m_{i}}, i=1,2$, are the unknown parameters.

The KL-optimality criterion is built according to Kullback-Leibler (KL) distance between the densities $f_{1}\left(y, x, \theta_{1}\right)$ and $f_{2}\left(y, x, \theta_{2}\right)$. If $f_{1}\left(y, x, \theta_{1}\right)$ is the true model and the alternative model is $f_{2}\left(y, x, \theta_{2}\right)$, the KL distance between $f_{1}\left(y, x, \theta_{1}\right)$ and $f_{2}\left(y, x, \theta_{2}\right)$ is

$$
\mathcal{D}\left(f_{1}, f_{2}, x, \theta_{2}\right)=\int f_{1}\left(y, x, \theta_{1}\right) \log \frac{f_{1}\left(y, x, \theta_{1}\right)}{f_{2}\left(y, x, \theta_{2}\right)} d y, \quad x \in \chi .
$$

The KL- optimality criterion function is given by

$$
I_{21}(\xi)=\min _{\theta_{2} \in \Omega_{2}} \int_{\chi} \mathcal{D}\left(f_{1}, f_{2}, x, \theta_{2}\right) \xi(d x) .
$$

A KL- optimum design maximizes $I_{21}(\xi)$ and is indicated by $\xi_{21}^{*}$.

The set

$$
\Omega_{2}(\xi)=\left\{\hat{\theta}_{2}: \hat{\theta}_{2}(\xi)=\arg I_{21}(\xi)\right\},
$$

is a singleton then the design is called a regular design, otherwise is called singular design. LópezFidalog et al. [9] proved that $\xi_{21}^{*}$ is a KL-optimum design if and only if $\psi_{21}\left(x, \xi_{21}^{*}\right) \leq 0, x \in \chi$, where, $\psi_{21}(x, \xi)=\mathcal{D}\left(f_{1}, f_{2}, x, \theta_{2}\right)-\int_{\chi} \mathcal{D}\left(f_{1}, f_{2}, x, \hat{\theta}_{2}\right) \xi(d x)$

is the directional derivative of Eq. (2).

For goodness of a design $\xi$ in the sense of discrimination purposes, the Kl-efficiency of a design $\xi$ relative to the optimum design $\xi_{21}^{*}$ is 


$$
\operatorname{Eff}_{21}(\xi)=\frac{I_{21}(\xi)}{I_{21}\left(\xi_{21}^{*}\right)}
$$

\subsection{C- optimum design}

Elfving [3] considered the geometric characterization of locally C-optimal designs. Obtaining C-optimal designs in linear and non-linear regression models have studied by many authors see for example, Studden [16]. C-optimality is based on estimating the linear combination of the parameters $c^{T} \beta$ with minimum variance, where $\mathrm{c}$ is a known vector of constants for more details see, Atkinson et al. [2] and Pazman and Pronzato [17].

The design criterion to be minimized is thus

$$
\operatorname{var} c^{T} \beta \propto c^{T} M^{-1}(\xi) c,
$$

where $\mathrm{c}$ is $p \times 1$. The equivalence theorem states that, for the optimum design,

$$
\left(\left\{f^{T}(x) M^{-1}\left(\xi^{*}\right) c\right\}^{2} \leq c^{T} M^{-1}\left(\xi^{*}\right) c, \quad x \in \chi .\right.
$$

For goodness of a design $\xi$, the C-efficiency of $\xi$ is defined as

$$
E f f^{C}(\xi)=\frac{c^{T} M^{-1}\left(\xi_{C}{ }^{*}\right) c}{c^{T} M^{-1}(\xi) c}
$$

\subsection{D-optimum design}

D-optimal designs are introduced to minimize the generalized variance of the estimated regression coefficients by maximizing the determinant of the Fisher information matrix. Mathematically,

$$
\max _{x_{i}, i=1, \ldots . ., n}\left|X^{T} X\right|=\min _{x_{i}, i=1, \ldots \ldots, n}\left|\left(X^{T} X\right)^{-1}\right| .
$$

D-optimal design has been central to work on optimum experimental designs and is studied widely; see Wang et al. [18].

For non-linear models, Silvey [19] defined the D-optimality criterion by

$\Phi_{D_{i}}\left[M_{i}\left(\xi, \theta_{i}\right)\right]=\left\{\begin{array}{cc}\log \left|M_{i}\left(\xi, \theta_{i}\right)\right| & \text { If } M_{i}\left(\xi, \theta_{i}\right) \text { is non }- \text { singular } \\ -\infty & \text { If } M_{i}\left(\xi, \theta_{i}\right) \text { is singular }\end{array}\right.$,

where $M_{i}\left(\xi, \theta_{i}\right)=\sum_{x \in \chi} J_{i}\left(x, \theta_{i}\right) \xi(x)$ is the information matrix corresponding to the statistical model $f_{i}\left(y, x, \theta_{i}\right)$ and $J_{i}\left(x, \theta_{i}\right)$ is the Fisher information matrix for a single observation on $y$ at $x, i=1,2$.

A design $\xi_{D_{i}}^{*}$ is a D-optimum design for the parameter estimation of the model $f_{i}\left(y, x, \theta_{i}\right)$ if and only if

$$
\psi_{D_{i}}\left(x, \xi_{D_{i}}^{*}\right) \leq 0, x \in \chi
$$

where, $\psi_{D_{i}}(x, \xi)=\operatorname{tr}\left[M_{i}^{-1}\left(\xi, \theta_{i}\right) J_{i}\left(x, \theta_{i}\right)\right]-m_{i}, \quad i=1,2$,

where $m_{i}$ is the number of the parameters in the model. 
The D-efficiency of a design $\xi$ is denoted by

$$
\operatorname{Eff}_{D_{i}}(\xi)=\frac{\left|M_{i}\left(\xi, \theta_{i}\right)\right|^{\frac{1}{m_{i}}}}{\left|M_{i}\left(\xi_{D_{i}}^{*}, \theta_{i}\right)\right|^{\frac{1}{m_{i}}}}, \quad i=1,2
$$

\section{3- Compound criteria}

A compound design criterion is defined as a weighted product of the efficiencies that is maximized to give an exact or a continuous design. As a beginning, two compound criteria will be proposed, which are DKL-optimality that combines D-optimality with KL-optimality for discriminating between models and CD-optimality that combines parameter estimation with Coptimality for feature of interest, respectively.

\subsection{DKL-optimum designs}

Tommasi [20] suggested DKL-optimality criterion for model discrimination and parameter estimation, which defined as a weighted geometric mean of KL- and D-efficiencies. The DKLcriterion can be used for any kind of regression models, nested or not. Hence, DKL-optimality criterion which is general applicability than DT-optimality criterion which introduced by Atkinson [21] applies only for nested regression models. When the assumed true model is $f_{2}\left(y, x, \theta_{2}\right)$ instead of $f_{1}\left(y, x, \theta_{1}\right)$, then the KL-optimum design is denoted by $\xi_{12}^{*}$.

The DKL-optimality criterion is defined by

$$
\Phi_{D K L}(\xi)=\left(\frac{I_{21}(\xi)}{I_{21}\left(\xi_{21}^{*}\right)}\right)^{\alpha_{1}}\left(\frac{I_{12}(\xi)}{I_{12}\left(\xi_{12}^{*}\right)}\right)^{\alpha_{2}}\left(\frac{\left|M_{1}(\xi, \theta)\right|}{\left|M_{1}\left(\xi_{D_{1}}^{*}, \theta\right)\right|}\right)^{\alpha_{3} / m_{1}}\left(\frac{\left|M_{2}(\xi, \theta)\right|}{\left|M_{2}\left(\xi_{D_{2}}^{*}, \theta\right)\right|}\right)^{1-\alpha_{1}-\alpha_{2}-\alpha_{3} / m_{2}},
$$

where, the coefficients $0 \leq \alpha_{i} \leq 1, i=1,2,3$, sum to one.

Taking logarithm in Eq. (10), the right-hand side becomes

$$
\begin{aligned}
\log \Phi_{D K L}(\xi)= & \alpha_{1} \log I_{21}(\xi)+\alpha_{2} \log I_{12}(\xi)+\alpha_{3} / m_{1} \log \left|M_{1}(\xi, \theta)\right|+ \\
& \frac{1-\alpha_{1}-\alpha_{2}-\alpha_{3}}{m_{2}} \log \left|M_{2}(\xi, \theta)\right| .
\end{aligned}
$$

A DKL-optimum design which maximizes $\log \Phi_{D K L}(\xi)$ is denoted by $\xi_{D K L}^{*}$. Following the general equivalence theorem for DKL-optimum which stated that the directional derivative of the criterion function Eq. (11) is $\psi_{D K L}\left(x, \xi_{D K L}^{*}\right) \leq 0$, where,

$$
\psi_{D K L}(x, \xi)=\alpha_{1} \frac{\psi_{21}(x, \xi)}{I_{21}(\xi)}+\alpha_{2} \frac{\psi_{12}(x, \xi)}{I_{12}(\xi)}+\frac{\alpha_{3}}{m_{1}} \psi_{D_{1}}(x, \xi)+\frac{1-\alpha_{1}-\alpha_{2}-\alpha_{3}}{m_{2}} \psi_{D_{2}}(x, \xi) .
$$

\subsection{CD-optimum design}

CD-optimality proposed by Atkinson et al. [2] which combining C-and D-optimality's criteria. CD-optimum design was applied for parameter estimation and estimation of the area under curve, which maximize a weighted product of the efficiencies

$$
\Phi_{C D}(\xi)=\left\{\frac{c^{T} M_{1}^{-1}\left(\xi_{c}^{*}\right) c}{c^{T} M_{1}^{-1}(\xi) c}\right\}^{\alpha_{4}}\left\{\frac{c^{T} M_{2}^{-1}\left(\xi_{c}^{*}\right) c}{c^{T} M_{2}^{-1}(\xi) c}\right\}^{\alpha_{5}}\left(\frac{\left|M_{1}(\xi, \theta)\right|}{\left|M_{1}\left(\xi_{D_{1}}^{*}, \theta\right)\right|}\right)^{\alpha_{6} / m_{1}}\left(\frac{\left|M_{2}(\xi, \theta)\right|}{\left|M_{2}\left(\xi_{D_{2}}^{*}, \theta\right)\right|}\right)^{1-\alpha_{4}-\alpha_{5}-\alpha_{6} / m_{2}}
$$


Taking logarthim in Eq. (13), the right hand side becomes

$$
-\alpha_{4} \log c^{T} M_{1}^{-1}(\xi) c-\alpha_{5} \log c^{T} M_{2}^{-1}(\xi) c+\frac{\alpha_{6}}{m_{1}} \log \left|M_{1}(\xi, \theta)\right|+\frac{1-\alpha_{4}-\alpha_{5}-\alpha_{6}}{m_{2}} \log \left|M_{2}(\xi, \theta)\right| .
$$

Designs maximizing Eq. (14) are called CD-optimum and are denoted by $\xi_{C D}^{*}$. The derivative function for Eq. (14) is given by

$$
\psi_{C D}(x, \xi)=\alpha_{4} \frac{\left\{f^{T}(x) M_{1}^{-1}(\xi) c\right\}^{2}}{c M_{1}^{-1}(\xi) c}+\alpha_{5} \frac{\left\{f^{T}(x) M_{2}^{-1}(\xi) c\right\}^{2}}{c M_{2}^{-1}(\xi) c}+\frac{\alpha_{6}}{m_{1}} \psi_{D_{1}}(x, \xi)+\frac{1-\alpha_{4}-\alpha_{5}-\alpha_{6}}{m_{2}} \psi_{D_{2}}(x, \xi) .
$$

Atkinson et al. [2] showed that the upper bound of $\psi_{C D}\left(x, \xi_{C D}^{*}\right)$ over $x \in \chi$ is one, achieved at the points of the optimum design.

\section{4- CDKL-optimum designs}

A new compound criterion CDKL-optimality will be constructed to estimate a parametric function such as the area under the curve, parameter estimation, and model discrimination for any kind of regression models.

To obtain CDKL-optimality criterion, a weighted geometric mean of efficiencies will be calculated $\left(\frac{I_{21}(\xi)}{I_{21}\left(\xi_{21}^{*}\right)}\right)^{\alpha_{1}}\left(\frac{I_{12}(\xi)}{I_{12}\left(\xi_{12}^{*}\right)}\right)^{\alpha_{2}}\left\{\frac{c_{1}{ }^{T} M_{1}^{-1}\left(\xi_{1}^{*}\right) c_{1}}{c_{1}{ }^{T} M_{1}^{-1}(\xi) c_{1}}\right\}^{\alpha_{3}}\left\{\frac{c_{2}{ }^{T} M_{2}^{-1}\left(\xi_{c_{2}}^{*}\right) c_{2}}{c_{2}{ }^{T} M_{2}^{-1}(\xi) c_{2}}\right\}^{\alpha_{4}}\left(\frac{\left|M_{1}(\xi, \theta)\right|}{\left|M_{1}\left(\xi_{D_{1}}^{*}, \theta\right)\right|}\right)^{\alpha_{5} / m_{1}}\left(\frac{\left|M_{2}(\xi, \theta)\right|}{\left|M_{2}\left(\xi_{D_{2}}^{*}, \theta\right)\right|}\right)^{1-\alpha_{1}-\alpha_{2}-\alpha_{3}-\alpha_{4}-\alpha_{5} / m_{2}}$

where, the coefficient $0 \leq \alpha_{i} \leq 1, i=1,2,3,4,5$, sum to one.

Taking logarithm in Eq. (16):

$\Phi_{C D K L}(\xi)=\alpha_{1} \log I_{21}(\xi)+\alpha_{2} \log I_{12}(\xi)-\alpha_{3} \log c_{1}{ }^{T} M_{1}^{-1}(\xi) c_{1}-$

$\alpha_{4} \log c_{2}{ }^{T} M_{2}^{-1}(\xi) c_{2}+\alpha_{5} / m_{1} \log \left|M_{1}(\xi, \theta)\right|+\frac{1-\alpha_{1}-\alpha_{2}-\alpha_{3}-\alpha_{4}-\alpha_{5}}{m_{2}} \log \left|M_{2}(\xi, \theta)\right|$.

A CDKL-optimum design, $\xi_{C D K L}^{*}$, maximizes $\Phi_{C D K L}(\xi)$. The directional derivative of the criterion function for Eq. (17) is given by:

$$
\begin{aligned}
& \psi_{C D K L}(x, \xi)=\alpha_{1} \frac{\psi_{21}(x, \xi)}{I_{21}(\xi)}+\alpha_{2} \frac{\psi_{12}(x, \xi)}{I_{12}(\xi)}+\alpha_{3} \psi_{c_{1}}(x, \xi)+\alpha_{4} \psi_{c_{2}}(x, \xi)+\frac{\alpha_{5}}{m_{1}} \psi_{D_{1}}(x, \xi)+ \\
& \frac{1-\alpha_{1}-\alpha_{2}-\alpha_{3}-\alpha_{4}-\alpha_{5}}{m_{2}} \psi_{D_{2}}(x, \xi) .
\end{aligned}
$$

The general equivalence theorem for CDKL criterion can be stated as follows: 
Theorem. For CDKL-optimal design, $\xi_{C D K L}^{*}$, the following conditions are equivalent;

i. A necessary and sufficient condition for a design $\xi_{C D K L}^{*}$ to be CDKL-optimum is fulfillment of the inequality $\psi_{C D K L}\left(x, \xi_{C D K L}^{*}\right) \leq 1, x \in \chi$, where the directional derivative $\psi_{C D K L}$ is given in Eq. (18).

ii. The upper bound of $\psi_{C D K L}\left(x, \xi_{C D K L}^{*}\right)$ is attained at the points of the optimum design.

iii. For any non-optimum design $\xi$, that is a design for which $\Phi_{C D K L}(\xi)<\Phi_{C D K L}\left(\xi_{C D K L}^{*}\right)$, $\sup _{x \in \chi} \psi_{C D K L}\left(x, \xi_{C D K L}^{*}\right)>1$.

\section{Proof.}

Since the terms in Equation (18) have been scaled, therefore the upper bound of $\psi_{C D K L}$ over $x \in \chi$ is one, achieved at the points of the optimum design. Moreover, $\psi_{C D K L}$ is the linear combination of the directional derivatives for C-optimality, D-optimality and KL-optimality criteria. Thus, the new criterion CDKL-optimality satisfies the conditions of convex optimum design and the theorem has been proved.

A measure of efficiency of a design $\xi$ relative to a CDKL-optimum design is given by

$$
E f f_{C D K L}(\xi)=\frac{\Phi_{C D K L}(\xi)}{\Phi_{C D K L}\left(\xi_{C D K L}^{*}\right)}
$$

\section{5- CDKL-optimum design for copula models}

Frèchet [22] introduced a question about the relationship between a multidimensional probability function and its lower dimensional margins, and Sklar [12] answered this question by using the copula function. The copula function ties the joint and the margins together. Thus, the instead of estimating the joint distribution we could be replaced by estimating the margins and constructing a copula.

Let $X_{i}=\left(X_{i,(1)}, X_{i,(2)}, \ldots \ldots, X_{i,(d)}\right)$ be i.i.d from some joint distribution, for which one or more parametric copula are employed. Letting $C_{\theta}(v)$ be such a copula, with density $c_{\theta}(v)$ on $[0,1]^{d}$, in terms of some parameter $\theta \in \Omega \subset \mathbb{R}^{d}$, the pseudo-log-likelihood function is

$$
l_{n}(\theta)=\sum_{i=1}^{n} \log c_{\theta}\left(\widehat{V}_{i}\right)
$$

In terms of the estimated and transformed marginal, say

$$
\widehat{V}_{i}=\left(\widehat{V}_{i,(1)}, \widehat{V}_{i,(d)}, \ldots \ldots, \widehat{V}_{i,(d)}\right)=\left(F_{n}^{(1)}\left(X_{i,(1)}\right), F_{n}^{(2)}\left(X_{i,(2)}\right), \ldots \ldots \ldots, F_{n}^{(d)}\left(X_{i,(d)}\right)\right),
$$

where, $F_{n}^{(i)}(x)=\frac{1}{n+1} \sum_{i=1}^{n} I\left\{X_{i}^{(i)} \leq x\right\}$.

In particular, the $\widehat{V}_{i}$ lie inside the unit square. The maximum $\hat{\theta}$ of $l_{n}($.$) is called the maximum$ pseudo-likelihood estimator, see GrØnneberg and Hjort [23]. 


\subsection{KL-criterion optimality for copula models}

Consider $A_{n}(\theta)=n^{-1} l_{n}(\theta)=n^{-1} \sum_{i=1}^{n} \log c_{\theta}\left(\widehat{V}_{i}\right)$. The pseudo-log-likelihood normalized by sample size. Suppose that the true data generating distribution has a density $f^{o}$. The unique decomposition

$$
f^{o}\left(x_{1}, x_{2}, \ldots \ldots, x_{d}\right)=c^{o}\left(F_{1}^{o}\left(x_{1}\right), F_{2}^{o}\left(x_{2}\right), \ldots \ldots, F_{d}^{o}\left(x_{d}\right)\right) \prod_{i=1}^{d} f_{i}^{o}\left(x_{i}\right) .
$$

$c^{o}$ is the true data known generating copula and $f_{i}^{o}$ is the true data generating marginal distributions.

There is a convergence almost obtained for each $\theta$ where,

$$
A_{n}(\theta) \underset{n \rightarrow \infty}{\longrightarrow} A(\theta)=\int_{[0,1]^{d}} c^{o}(v) \log c_{\theta}(v) d v
$$

Let $\theta^{o}$ is the least false value for the family, i.e. the maximizer of $A($.$) , assumed to be$ unique and an inner point of the parameter space. This is also the minimizer of the KullbackLeibler distance (KL-distance).

In order to discriminate between the parametric and true copula $c_{\theta}, c^{o}$, use Kl-optimality criterion for copula which defined by

$$
I_{\theta o}(\xi)=\min _{\theta \in \Omega} \int_{[0,1]^{d}} K L\left(c^{o}, c_{\theta}\right) \xi d(v),
$$

where, $K L\left(c^{o}, c_{\theta}\right)=\int_{[0,1]^{d}} c^{o} \log \frac{c^{o}}{c_{\theta}} d v$ is the Kullback-Leibler distance for the parametric and true copula $c_{\theta}, c^{o}$. A KL-optimum design maximizes $I_{\theta o}(\xi)$.

\subsection{C-, D-criterion optimality for copula models}

The corner stone for defining the D- and C-optimality criteria for copula, development of the information matrix is provided to match copula models. It was discussed by many papers see for example, GrØnneberg and Hjort [23] and Perrone and Müller [24].

The information matrix for copula is defined by

$$
\mathcal{J}=\mathbb{E}_{\phi}\left(\xi, \theta_{o}\right) \phi\left(\xi, \theta_{o}\right)^{t},
$$

where, $\phi(., \theta)=\partial / \partial \theta \log c(., \theta)$ and $\xi \sim C^{o}$.

To apply CDKL-optimality criterion for copula models for instance, on product, Gaussian, Farlie-Gumbel-Morgenstern (FGM), Clayton, Frank and Gumbel copula models, equations (20) and (21) besides the equivalence theorem in Section 4 (Theorem) are provided.

\section{References}

[1] K. Smith, "On the standard deviations of adjusted and interpolated values of an observed polynomial function and it's constant and the guidance they give towards a proper choice of the distribution of observations". Biometrika, 1918, 12, 1-85.

[2] A. Atkinson, A. Donev, and R.Tobias, "Optimum experimental designs, with SAS". 2007, Oxford university press, New York. 
[3] G. Elfving, "Optimum allocation in linear regression theory". Annals of Mathematical Statistics, 1952, 23, 255-262.

[4] H. Dette and T. Holland-Letz, "A geometric characterization of C-optimal designs for heteroscedastic regression". The Annals of Statistics, 2009, 37,6B, 4088-4103.

[5] A. Wald, "On the efficient design of statistical investigations". Annals of Mathematical Statistics, 1943, 14, 134-140.

[6] A. Atkinson and V. Fedorov, "The design of experiments for discriminating between two rival models". Biometrika, 1975a, 62 (1), 57-70.

[7] A. Atkinson and V. Fedorov, "Optimal design: experiments for discriminating between several models". Biometrika, 1975b, 62(2), 289-303.

[8] J. López-Fidalgo, C. Tommasi, and P. Trandafir, "Optimal designs for discriminating between heteroscedastic models". In: Proceedings of the 5th St. Petersburg Workshop on Simulation, NII Chemistry Saint Petersburg University Publishers, Saint Petersburg, 2005, 429-436.

[9] J. López-Fidalog, C. Tommasi and P. Trandafir, "An optimal experimental design criterion for discriminating between non-normal models”. Journal of the Royal Statistical Society, B., 2007, 69, 231-242.

[10] C. Tommasi, "Optimal designs for discriminating among several non-Normal models". In: López-Fidalgo, J., Rodrìguez-Dìaz, J.M., Torsney, B. (Eds): MODA 8 - Advances in ModelOriented Design and Analysis. Physica-Verlag, 2007, 213-220.

[11] M. Abd El-Monsef and M. Seyam, "CDT-optimum designs for model discrimination, parameter estimation and estimation of a parametric function”. Journal of Statistical Planning and Inference, 2011,141, 639-643.

[12] A. Sklar, "Fonctions de repartition à $n$ dimensions et leur smarges". Publications de l'Institutde Statistique de l'Université de Paris, 1959, 8, 229-231.

[13] A. Nikoloulopoulos and D. Kalis, "Multivaraite logit copula model with an application to dental data". Statistics Medicine, 2008, 27(30), 6393-6406.

[14] P. Danaher and M. Smith, "Modeling multivariate distributions using copulas: applications in marketing". Marketing Science, 2011, 30 (1), 4-21.

[15] A. Patton, "A review of copula models for economic time series". Journal of Multivariate Analysis, 2012, 110, 4-18.

[16] W. Studden, "Elfving's theorem revisited". Journal of Statistical Planning and Inference, 2005,130, 85-94. MR2127757.

[17] A. Pazman and L. Pronzato, "Asymptotic normality of nonlinear least squares under singular experimental designs. In optimal design and related areas in optimization and statistics”, 2009, Springer, Heidelberg.

[18] Y. Wang, R. Myers , E. Smithand and K. Ye, (2006). "D-optimal designs for Poisson regression models". Journal of Statistical Planning and Inference, 136, 2831-2845.

[19] S. Silvey, “Optimal design”. 1980, Chapman \& Hall. London.

[20] C. Tommasi, "Optimal designs for both model discrimination and parameter estimation". Journal of Statistical Planning and Inference, 2009, 139, 4123-4132.

[21] A. Atkinson, "DT-optimum designs for model discrimination and parameter estimation". Journal of Statistical planning and Inference, 2008, 138, 56-64.

[22]M. Fréchet, "Sur les tableaux de correlation dont les marges sont données". Ann University Lyon Sect A, 1951, 9, 53-77.

[23] S. GrØnneberg and N. Hjort, "The copula information criterion”. Statistical research report, 2008, 7. Dep. of Math. University of Oslo.

[24] E. Perrone and W. Müller, "Optimal designs for copula models". Statistics: A journal of Theoretical and Applied Statistics, 2016, 50(4). 